\title{
Acute stress show great influences on liver function and the expression of hepatic genes associated with lipid metabolism in rats
}

\author{
Xiaoling Gao, Yuaner Zeng, Shuqiang Liu and Shuling Wang*
}

\begin{abstract}
Background: The theory of Chinese medicine believes rage harms normal liver function, namely 'raged impairing liver' in short. The purpose of this study is to investigate the impact of acute stress on liver lipid metabolism in rats.

Methods and results: Comparison of liver function indicators, serum lipid level of rats under acute stress and normal rats, as well as detection of liver tissue in the SR - BI, ABCG5 and ABCG8 protein and gene expression changes. Acute stressed rats had shown a lower serum levels of albumin $(P<0.01)$, HDL- cholesterol $(P<0.01)$ than normal rats, with higher serum levels of globulin $(P<0.01)$ and LDL-cholesterol $(P<0.05)$. Acute stressed rat's liver tissue exhibited a lower protein expression of ABCG5 $(P<0.05)$, ABCG8 $(P<0.01)$ and a higher level of $\mathrm{SR}-\mathrm{BI}(P<0.05)$, compared with to normal rats. Furthermore, liver gene expression of ABCG5 $(P<0.01)$ and $A B C G 8(P<0.05)$ were lower in acute stressed rats than in normal rats, while SR-BI was higher in acute stressed rats than in normal rats $(P<0.01)$.
\end{abstract}

Conclusions: Acute stress had a direct influence on rat's liver lipid metabolism.

Keywords: Acute stress, Lipid metabolism, Raged impairing liver, Scavenger receptor BI, ATP-binding cassette transporter

\section{Background}

The word 'stress' first appeared in the index of Psychological Abstracts in 1944 [1]. Recently, stress has shown to play an important role in the onset of cardiovascular diseases, immunological disorders and pathophysiological consequences of normal aging [2-4]. Stress had also been shown that will inhibit metabolic function, such as lipid elimination and glycometabolism [5,6]. The theory Chinese Medicine believes rage harms liver function [7]. Meanwhile, acute stress causes rage, so acute stress may individually damages the liver. There are reports [8-11] that acute stress increases oxidation and diminishes antioxidant's protection, stating acute stress oxidates and destroys the liver. The liver is a crucial organ that facilitate lipid metabolism, including cholesterol metabolism and it is meaningful to study acute stress's effect on lipid metabolism in rats. In recent years,

\footnotetext{
* Correspondence: jingelwang@gzucm.edu.cn

School of Chinese Pharmaceutical Science, Guangzhou University of Chinese Medicine, Guangzhou, Guangdong, China
}

the study of lipid metabolism focused on reverse cholesterol transport (RCT), which means excess cholesterol transport from peripheral cells of apolipoprotein to the liver by high density lipoprotein (HDL), where lipid uptake took place and cleared. ATP-binding cassette transporter $(\mathrm{ABC}) \mathrm{G5}, \mathrm{G8}$ and scavenger receptor- BI (SR-BI) are principal mediators of cholesterol efflux [12-14]. ABCG5 and ABCG8 mediate cholesterol outflow from peripheral cells, while SR-BI mediate hepatic selective uptake of cholesterol esters. Present study aims to discuss the effect of acute stress on lipid metabolism in rats from the perspective of reverse cholesterol transport.

\section{Materials and methods \\ Animals}

Adult male Sprague Dawley rats weighing 160-180 g were obtained from the Central Animal Facility, Guangzhou University of Chinese Medicine (SCXK (Guangdong) 2008-0020), the rats were housed individually per cage upon arrival, they were allowed to adjust to their new environment in 1 week. The rat had free access to water and 
food, temperature of $25 \pm 1^{\circ} \mathrm{C}$, a 12-hour light-dark cycle (lights on at 08:00-20:00). Protocols were conducted in accordance with standard ethical guidelines, it was approved by the institution animal ethics committee (Grants 81102530/H2702 from National Natural Science Foundation of China). The rats were randomly selected into normal group and acute stress group $(n=8$ in each group). Rats in the acute stress group were subjected to tail clipping at one third towards the end of the tail, three times a day for 30 minutes each time [15]. The normal control group were housed in a separate room and were not subjected to any stress for 14 consecutive days. Collection of blood, sacrifice of rats and liver isolation were performed after the restraint stress procedure.

\section{Sucrose consumption test}

All rats were given $1 \%$ sucrose solution for a 24 hour period in their cages with no food and water following food and water deprivation for 24 hours. Sucrose consumption was recorded by re-weighing bottles of solution.

\section{Open-field test}

Open-field test was conducted as described by Dunn [16], to assess acute stress effects on rats, the test was performed on the last 2 days before the end of the experiment. The apparatus consists of a rectangular box $(80 \times 80 \times 50 \mathrm{~cm})$, a floor divided into $25(16 \times 16 \mathrm{~cm})$ rectangular units. The rats were placed in the center of an open field and were allowed to freely explore the area for 3 minutes. Two motor parameters were quantified throughout this test: locomotion frequency (numbers of with which the rat crossed one of the grid lines with all four paws) and rearing frequency (times of rearing with hind legs). The animals were acclimated to the experimental room for at least 2 hours prior to the beginning of the open-field test. Open-field tests were carried out in soundproof rooms without any human interference. The open-field was cleaned with a 5\% water-ethanol solution before behavioral testing to eliminate bias due to odors left by previous rats. The evaluation of rat's motivation behavior was performed in a blinded manner by two independent observers. After testing inter-observer reliability, the mean from the results was statistically analyzed.

\section{Biochemical analysis}

Blood samples were collected under chloral hydrate anaesthesia and centrifuged at $3500 \mathrm{rpm}$ for 15 minutes, serum was separated at $4^{\circ} \mathrm{C}$ by a refrigerated centrifuge (HEMA, China). Serum concentrations of total cholesterol (TC), total triglyceride (TG), low-density lipoprotein cholesterol (LDL-C), high-density lipoprotein cholesterol (HDL-C), alanine aminotransferase (ALT), aspartate aminotransferase (AST), albumin (ALB) and globulin (GLB) were measured by enzymatic assays using an automated biochemical analyzer (Cobas 8000, Roche, Germany).

\section{Quantitative real-time PCR}

Total RNA was extracted with Trizol reagent (Invitrogen, Shanghai, China). The quality of samples was confirmed by agarose gel electrophoresis. Total RNA was reverse transcribed to complementary DNA using Prime Script ${ }^{\circ}$ 1st Strand cDNA Synthesis Kit (TAKARA, Kyoto, Japan). The thermo cycler settings were $30^{\circ} \mathrm{C}$ for 10 minutes, $42^{\circ} \mathrm{C}$ for 60 minutes, and $70^{\circ} \mathrm{C}$ for 15 minutes. Real-time PCR was performed in triplicates using SYBR Premix Ex Taq kit (TAKARA) in a $25 \mu \mathrm{L}$ reaction volume. GAPDH was used as the housekeeping gene. The primers used for real-time PCR were listed in Table 1. Relative mRNA levels were calculated by the method of $2^{-\Delta \Delta^{\mathrm{Ct}}}$.

\section{Western blot analysis}

Tissue samples were frozen in liquid nitrogen. Total protein was isolated in a lysis buffer, resolved by $10 \%$ odium dodecyl sulfate polyacrylamide gel electrophoresis (SDS-PAGE) and transferred onto polyvinylidene fluoride (PVDF) membranes by electroblotting. SR-BI, ABCG5 and ABCG8 proteins were detected using an anti- SR-BI monoclonal antibody (1:500 dilution, Abcam, UK), antiABCG5 polyclonal antibody (1:500 dilution, Bioss, Beijing, China), or anti-ABCG8 polyclonal antibody (1:500 dilution, Bioss, Beijing, China). The bands were visualized with an enhanced chemiluminescence kit (Millipore, Billerica, MA) and analyzed with Image-Pro Plus 6.0 (Media Cybernetics, US).

\section{Statistical analysis}

Each parameter was expressed as the mean \pm SEM. Statistical significance was determined by t-Student's test. A $p$ value of less than 0.05 was considered significant.

\section{Results}

\section{Sucrose consumption test}

Compared with the activity of sucrose consumption of normal group, $2.36 \pm 0.15$, the activity of sucrose consumption

Table 1 Primers used for real-time PCR analysis

\begin{tabular}{lll}
\hline Gene & Primer & Sequence $\left(\mathbf{5}^{\prime} \boldsymbol{\rightarrow} \mathbf{3}^{\prime} \mathbf{)}\right.$ \\
\hline SR-BI & Sense & CCCCATGAACTGTTCCGTGA \\
& Anti-sense & CCACAGCAATGGCAGGACTA \\
ABCG5 & Sense & GGGAAGTGTTTTGAACGGC \\
& Anti-sense & GTGTATCTCAGCGTCTCCCG \\
ABCG8 & Sense & ACGTGGACTTGACGAGCATT \\
& Anti-sense & GTGTCCTGTGTGAGGGTCTG \\
GAPDH & Sense & TGCTGGGGCTGGCATTGCTCTC \\
& Anti-sense & ATGAGGTCCACCACCCTGTGC \\
\hline
\end{tabular}


of model group, $2.06 \pm 0.25$, is much lower than compared with the activity of sucrose consumption the normal group, which states notable statistic difference $(P<0.05)$ (Figure 1$)$.

\section{Acute stress exposure resulted in depression-like symptoms}

Compared to the activities of the horizontal and the vertical of normal group, which $84 \pm 9$ and $20 \pm 4$, the activities of the horizontal and the vertical of model group were much lower, the results of these two indexes were $56 \pm 15$ and $13 \pm 5$. We can find remarkable statistical difference $(P<0.01$ or $P<0.05)$ (Figure 2$)$.

\section{Body weight and liver coefficient}

As shown in Figure 3A, during the control phase (baseline), all animals (normal and model groups) exhibited a similar weight, $180.7 \pm 8.1 \mathrm{~g}$ and $178.1 \pm 10.0 \mathrm{~g}$. However, after two weeks of acute stress, reduced this preference in the model group, which are $183.0 \pm 8.6 \mathrm{~g}$ and $202.2 \pm$ $14.5 \mathrm{~g}$, the body weight of normal group in the next were $229.7 \pm 8.5 \mathrm{~g}$ and $256.8 \pm 14.7 \mathrm{~g}$, the differences between two groups extremely remarkable difference $(P<0.01)$ (Figure 3A). As shown in Figure 3B, two weeks of acute stress decrease the relative liver coefficient (weight/100 g body weight), $3.16 \pm 0.12$, the liver coefficient of normal group, $3.43 \pm 0.18$, is much higher as compared with the liver coefficient of model group, which states notable statistic difference $(P<0.01)$ (Figure 3B).

\section{Comparison of serum and blood lipid metabolism index between each group}

The level of TC, TG, LDL-C and HDL-C of the normal rats were $1.56 \pm 0.14 \mathrm{mmol} / \mathrm{L}, 0.46 \pm 0.14 \mathrm{mmol} / \mathrm{L}, 0.22 \pm$

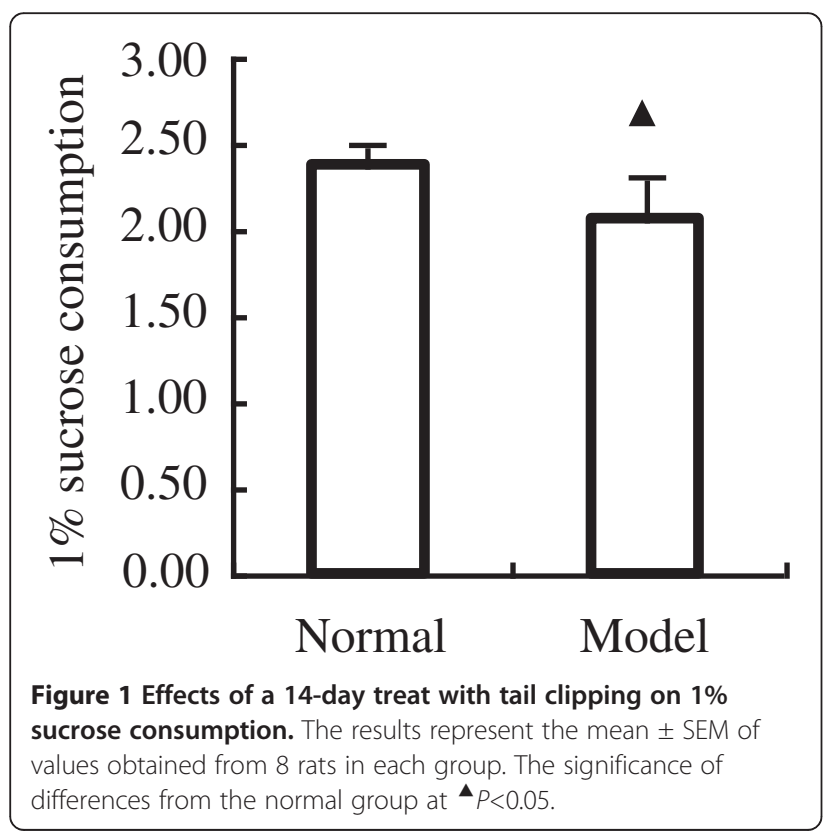

$0.03 \mathrm{mmol} / \mathrm{L}, 0.73 \pm 0.06 \mathrm{mmol} / \mathrm{L}$, comparing to the normal group, the rats have a higher level of $\mathrm{TC}, 1.61 \pm$ $0.12 \mathrm{mmol} / \mathrm{L}$, higher level of TG, $0.47 \pm 0.14 \mathrm{mmol} / \mathrm{L}$, but shown no statistical differences $(P>0.05)$, a clear higher level of LDL-C, $0.30 \pm 0.07 \mathrm{mmol} / \mathrm{L}$, a lower level of HDL$\mathrm{C}$ at $0.61 \pm 0.05 \mathrm{mmol} / \mathrm{L}$, which show remarkable statistic differences $(P<0.05$ or $P<0.01)$ (Table 2$)$.

\section{Comparison of serum liver function index between each group}

Compared with the activities of ALB, GLB, ALT and AST of normal group, which are $38.9 \pm 0.8 \mathrm{~g} / \mathrm{L}, 21.3 \pm 1.1 \mathrm{~g} / \mathrm{L}$, $43 \pm 12 \mathrm{U} / \mathrm{L}$ and $97 \pm 15 \mathrm{U} / \mathrm{L}$, respectively, the rats in model group has a lower level of ALB, $34.8 \pm 2.8 \mathrm{~g} / \mathrm{L}$, a higher level of GLB, $23.0 \pm 1.0 \mathrm{~g} / \mathrm{L}$, the difference between these two groups were statistically significant $(P<0.01)$. The level of ALT and AST of the rats in model group were separate at $41 \pm 10 \mathrm{U} / \mathrm{L}$ and $105 \pm 29 \mathrm{U} / \mathrm{L}$, comparing to the index of normal group, it illustrate no statistical differences $(P>0.05)$ (Table 3$)$.

\section{Acute stress affects lipid metabolism in rats}

We examined the effects of acute stress on expression levels of liver ABCG5, ABCG8 and SR-BI using western blotting. As shown in Figure 4B, the expressions of ABCG5 and ABCG8 of all of these livers were lower in model rats, while SR-BI was higher. These results were confirmed by quantitative PCR assays showing that messenger RNA (mRNA) levels of ABCG5 and ABCG8 were all down-regulated in model rats (Figure 4A) whereas SR-BI mRNA seemed to be up-regulated.

\section{Discussion}

Anger is one of the seven emotions in Traditional Chinese medicine (TCM). Chinese medicine theory believes raged harms normal liver function, 'raged impairing liver'. SU WEN . The disease theory, wrote "In great anger, an increase in $Q i$ damages the liver." Meanwhile, $S U$ WEN . Theory of Yin and Yang, wrote "anger, as liver emotion", the so-called anger is metaplasia by the vital essence. If anger hurt viscera, it hurts the born of the viscera.

Stress was put forward by Han Salye in 1944, it is a kind of syndrome reaction state including nerve, endocrinology and immunity, when the body faces strong stimulus from the outside world. When confronting stressors, the hypothalamic-pituitary-adrenal axis is excited during stress, the adrenal endocrine releases more glucocorticoids, meanwhile, the glucose level in blood is also higher, and the immunity function is depressed. Good stress reaction is beneficial to the body in a short term environment, if the body under stress for an extended time, the HPA axis is over excited, GC level is too high, then the body is hurt. Stress has been shown to play an important role in the onset of 

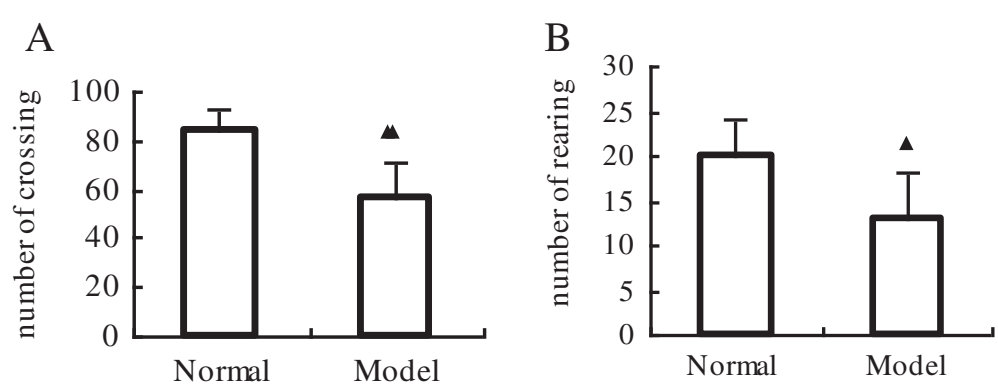

Figure $\mathbf{2}$ Comparison of behavioral motor effects elicited by acute stress in open-field test. A, Horizontal activity estimated as the number of grids crossed during a 3-minute test. $\mathbf{B}$, The frequency of rearing with hind legs during a 3-minute test. The data were obtained one day before sacrificed. Data are expressed as mean \pm SEM $(n=8) . \quad{ }^{\mathbf{A}} P<0.05$ and $\boldsymbol{\Delta} \mathbf{\Delta} P<0.01$ compared with the normal group.

cardiovascular disease [17-20], it has also been found to inhibit metabolic functions such as lipid elimination and glycometabolism [5,6].

There have studies [8-11] reported that stress has some influences on the liver, such as oxidative stress. According to Chinese medicine, rage harms the liver, since liver plays a great role in lipid metabolism, studying the impact of acute stress on lipid metabolism helps to explain the theory. This study established a raged-rat model (refers to a method described in Experiment of Zoology, issued by Science Press, China) by clipping its tail, three times a day for 30 minutes each time. In the preliminary experiments, we found that the method mentioned in Experiment of Zoology caused huge damages to rats, we determined to adjust this tail clipping to three times a day. We found acute stress affected the behaviors of rats, which is proved in the open-field test, a method used to evaluate the behavior of animal. The horizontal scores of open-field test reflects the excitability of rats, while vertical scores reflects the rat's to the environment $[21,22]$. Both the activities of the model group are lower than the normal group, the result shows excitability and exploration activities of rats decreased due to being subjected to stress.
With normal rats, we found serum ALB level was lower in the model group, with higher GLB, LDL-C and lower HDL-C, demonstrated acute stress may damage rat's liver, leading to a reduction in ALB production, an increase in GLB. ALB is manufactured in the liver, when liver is impaired, it decreases the production of ALB. At the same time, GLB is manufactured by immune organ, when there is an 'enemy' in the body, GLB increase in production. Serum concentrations of HDL-C have strong inverse correlations with the risk of cardiovascular disease, independently of LDL-C levels, indicate that acute stress may influencing the HDL-C and LDL-C levels of serum, thus affecting the liver lipid metabolism. Due to the stress of time is not long or strong enough, the extent of the damage of liver cells is not particularly serious. So there are no significant differences in ALT and AST levels between the normal group and model group in the present study.

In this study, the liver tissue of model group exhibited a lower gene expression of ABCG5, ABCG8 and a higher SR-BI level, when compared to normal rats. Furthermore, liver protein expression of ABCG5 and ABCG8 were lower in the model group than the normal group, while SR-BI was higher in the model group than the
A

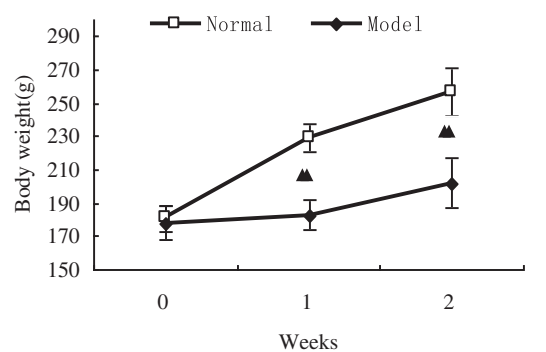

\section{B}

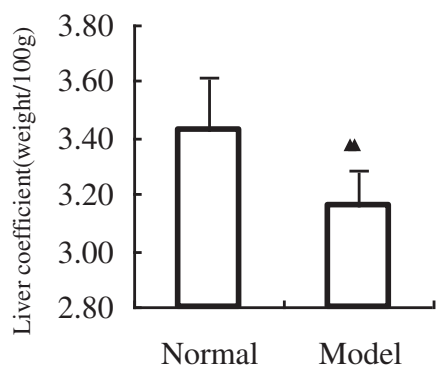

Figure 3 Effect of acute stress on body weight and liver coefficient in rats. A, Results of rat's weight of submitted after 2 weeks of acute stress. B, Results of the liver coefficient of rats submitted after 2 weeks of acute stress. Data are expressed as mean $\pm \operatorname{SEM}(n=8)$. $\mathbf{\Delta} \mathbf{\Delta} P<0.01$ versus the normal group. 
Table 2 Comparison of serum lipid metabolism index between each group

\begin{tabular}{lccccc}
\hline & \multirow{2}{*}{$\begin{array}{c}\text { Control } \\
(\mathbf{n}=\mathbf{8})\end{array}$} & $\begin{array}{c}\text { Model } \\
(\mathbf{n}=\mathbf{8})\end{array}$ & \multicolumn{3}{c}{ T-test results } \\
\cline { 3 - 6 } & & $\mathbf{t}$ & $\mathbf{d f}$ & $\mathbf{s i g}$ \\
\hline $\mathrm{TC}(\mathrm{mmol} / \mathrm{L})$ & $1.56 \pm 0.14$ & $1.61 \pm 0.12$ & -0.841 & 14 & 0.415 \\
$\mathrm{TG}(\mathrm{mmol} / \mathrm{L})$ & $0.46 \pm 0.14$ & $0.47 \pm 0.14$ & -0.142 & 14 & 0.889 \\
$\mathrm{LDL}(\mathrm{mmol} / \mathrm{L})$ & $0.22 \pm 0.03$ & $0.30 \pm 0.07 \mathbf{\Delta}$ & -2.916 & 14 & 0.011 \\
$\mathrm{HDL}(\mathrm{mmol} / \mathrm{L})$ & $0.73 \pm 0.06$ & $0.61 \pm 0.05 \mathbf{\Delta}$ & 4.57 & 14 & 0.000 \\
\hline
\end{tabular}

Data are presented as mean \pm SEM. ${ }^{\mathbf{\Delta}} P<0.05$ and ${ }^{\mathbf{} \wedge} P<0.01$ compared with the normal group.

normal group. ABCG5 and ABCG8 are ATP-binding cassette transporters, which expressed mainly in the liver and small intestine, mediated lipid membrane unidirectional outflow into the extracellular. After acute stress applied, ABCG5 and ABCG8 decreased expression in the liver, henced the intracellular lipid efflux was suppressed. The results show that, acute stress may affect the liver lipid efflux from the cells, thereby affecting the normal operation of the lipid metabolism, and may also be one of the reasons the Chinese say 'raged impairing liver'. SR-BI is one kind of HDL receptors, first determined at the molecular level, having highly binding force with HDL, and mediated hepatic selective uptake of cholesterol ester. Increased the expression of liver SR-BIcan be effective in promoting reverse cholesterol transport. But in this study, SR-BI expression increased after acute stress was applied. This may be in the early acute stress process, mature HDL decreased in production, SR-BI expression in the liver cell membrane transient up-regulation in order to better capture of HDL. Specific mechanism needs to be followed up in future studies.

\section{Conclusions}

In conclusion, we confirmed here for the first time acute stress decreased the expression of hepatic ABCG5 and ABCG8 besides up-regulated SR-BIin raged rats. Our data shown that acute stress harms normal liver functions, affects the level of lipid metabolism. Elevated serum GLB and LDL-C levels, reduced serum ALB and

Table 3 Comparison of serum liver function index between each group

\begin{tabular}{lccccc}
\hline & \multirow{2}{*}{$\begin{array}{c}\text { Control } \\
(\mathbf{n}=\mathbf{8})\end{array}$} & $\begin{array}{l}\text { Model } \\
(\mathbf{n}=\mathbf{8})\end{array}$ & \multicolumn{3}{c}{ T-test results } \\
\cline { 4 - 6 } & & & $\mathbf{t}$ & $\mathbf{d f}$ & $\mathbf{s i g}$ \\
\hline $\mathrm{ALB}(\mathrm{g} / \mathrm{L})$ & $38.9 \pm 0.8$ & $34.8 \pm 2.8^{\mathbf{\Delta}} \mathbf{\Delta}$ & 3.896 & 14 & 0.002 \\
$\mathrm{GLB}(\mathrm{g} / \mathrm{L})$ & $21.3 \pm 1.1$ & $23.0 \pm 1.0^{\mathbf{\Delta}}$ & -3.222 & 14 & 0.006 \\
$\mathrm{ALT}(\mathrm{U} / \mathrm{L})$ & $43 \pm 12$ & $41 \pm 10$ & 0.33 & 14 & 0.745 \\
$\mathrm{AST}(\mathrm{U} / \mathrm{L})$ & $97 \pm 15$ & $105 \pm 29$ & -0.77 & 14 & 0.453 \\
\hline
\end{tabular}

Data are presented as mean \pm SEM. ${ }^{\mathbf{\Lambda}} P<0.01$ compared with the normal group.

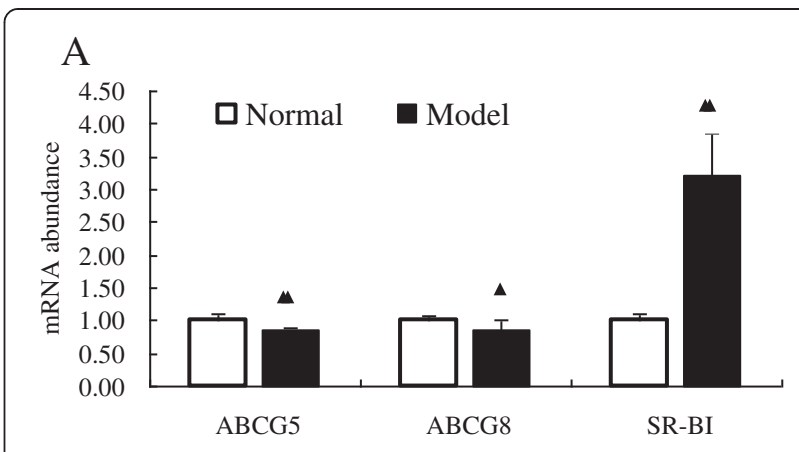

B

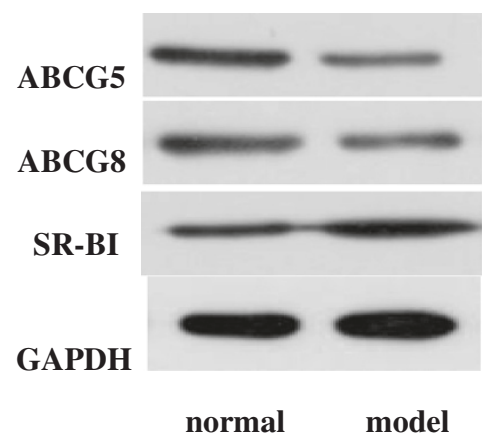

Figure 4 Acute stress affects lipid metabolism was associated with the inhibition of ABCG5, ABCG8 and SR-BI. A: The expression pattern of messenger RNA (mRNA) of ABCG5, ABCG8 and $S R-B I$ in the liver measured with quantitative polymerase chain reaction. B: Western blots illustrate acute stress decreased ABCG5 and $A B C G 8$, while increased SR-BI. Data are presented as mean \pm SEM ( $n=8) .{ }^{\mathbf{\Delta}} P<0.05$ and $\boldsymbol{\Delta} \boldsymbol{\Delta} P<0.01$ versus the normal group, t-test, ( $A B C G 5: t=3.597 ; A B C G 8: t=2.61 ; S R-B I: t=-9.335)$.

HDL-C levels, inhibited of reverse cholesterol transporter gene expression may all contribute to the harmful effects of acute stress. Our findings help to explain the Chinese medicine terminology of raged impairing liver.

\section{Abbreviations}

ALT: Alanine aminotransferase; AST: Aspartate aminotransferase;

ALB: Albumin; GLB: Globulin; TC: Total cholesterol; TG: Total triglyceride; HDL-C: High-density lipoprotein cholesterol; LDL-C: Low-density lipoprotein cholesterol; SR-BI: Scavenger receptor Bl; ABCG: ATP-binding cassette transporter.

\section{Competing interests}

The authors declare that they have no competing interests.

\section{Authors' contributions}

XLG participated in the design of the study, and performed statistical and genetic analysis and drafted the manuscript. SQL carried out by the Western blotting. YEZ performed blood samples analysis. SLW conceived of the study, and participated in its design and coordination and helped to draft the manuscript. All authors read and approved the final manuscript.

\section{Acknowledgements}

This work was supported by Grants $81102530 / \mathrm{H} 2702$ from National Natural Science Foundation of China, it was supported by the Laboratory System of Guangdong Province. We wish to thank the members of Guangdong Metabolic Disease Lab and other departments of Guangzhou University of Chinese Medicine who contributed to this study. Finally, we are grateful to Guangdong Provincial Hospital of Traditional Chinese Medicine for the 
technical services in biochemical detection, and show great thanks to Guangzhou Vipotion Biotechnology Co., Ltd. for their technical assistants in RT-PCR and Western blotting.

Received: 5 June 2013 Accepted: 29 July 2013

Published: 31 July 2013

\section{References}

1. Esch T: Health in stress: change in the stress concept and its significance for prevention, health and life style. Gesundheitswesen 2002, 64:73-81.

2. Esch T, Stefano GB, Fricchione GL, Benson H: Stress in cardiovascular diseases. Med Sci Monit 2002, 8:93-101.

3. Chen WW, He RR, Li YF, Li SB, Tsoi B, Kurihara H: Pharmacological studies on the anxiolytic effect of standardized Schisandra lignans extract on restraint-stressed mice. Phytomedicine 2011, 18:1144-1147.

4. He RR, Yao XS, Li HY, Dai Y, Duan YH, Li YF, Kurihara H: The anti-stress effects of Sarcandra glabra extract on restraint-evoked immunocompromise. Biol. Pharm. Bull 2009, 32:247-252

5. Gopaul NK, Manraj MD, Hebe A, Lee Kwai Yan S, Johnston A, Carrier MJ, Anggard EE: Oxidative stress could precede endothelial dysfunction and insulin resistance in Indian Mauritians with impaired glucose metabolism. Diabetologia 2001, 44:706-712.

6. Gigon A, Matos AR, Laffray D, Zuily-Fodil Y, Pham-Thi AT: Effect of drought stress on lipid metabolism in the leaves of Arabidopsis thaliana. Ann Bot 2004, 94:345-351.

7. Gonzalez-Flecha B, Cutrin JC, Boveris A: Time cource and mechanism of oxidative stress and tissue damage in rat liver subjected to in vivo ischemia-reperfusion. J Clin Invest 1993, 91:456-464.

8. Mittler R: Oxidative stress, antioxidants and stress tolerance. Trends Plant Sci 2002, 7:405-410.

9. Berlett BS, Stadman ER: Protein oxidation in aging, disease, and oxidative stress. J Biol Chem 1997, 272:20313-20316.

10. Pu HJ, Cao YF, He RR, Zhao ZL, Song JH, Jiang B, Huang T, Tang SH, Lu JM, Kurihara H: Correlation between Anti-stress and Hepatoprotective Effects of Schisandra Lignans Was Related with Its Anti-oxidative Actions in Liver Cells. Evidence-Based Complementary and Alternative Med 2012, 2012:161062.

11. Zhai YJ, He RR, Tsoi B, Li YF, Li XD, Tsuruoka N, Abe K, Kurihara H: Protective effect of extract of chicken meat on restraint stress-induced liver damage in mice. Food Function 2012, 3:662-667.

12. Ye D, Lammers B, Zhao Y, Meurs L, Van Berkel TJ, Van Eck M: ATP-binding cassette transporters $\mathrm{A} 1$ and $\mathrm{G1}, \mathrm{HDL}$ metabolism, cholesterol efflux, and inflammation: important targets for the treatment of atherosclerosis. Current Drug Targets 2011, 12:647-660

13. Hoekstra M, Van Berkel TJ, Van eck M: Scavenger receptor BI: a multipurpose player in cholesterol and steroid metabolism. World J Gastroenterol 2010, 16:5916-5924

14. Fabre AC, Malaval C, Ben Addi A, Verdier C, Pons V, Serhan N, Lichtenstein L, Combes G, Huby T, Briand F, Collet X, Nijstad N, Tietge U, Robaye B, Perret $B$, Boeynaems JM, Martinez LO: P2Y13 receptor is critical for reverse cholesterol transport. Hepatology 2010, 52:1477-1483.

15. Zou YH, Xu ZW, Su GQ: Animal models of human diseases: the experiment of zoology: chapter 7. In 6th edition Edited by Zou YH. China: Science Press; 2010:140.

16. Dunn AJ, Swiergiel AH: Effects of interleukin-1 and endotoxin in the forced swim and tail suspension tests in mice. Pharmacol Biohem Behav 2005, 81:688-693.

17. Bassenge $E$, Schneider HT, Daiber A: Oxidative stress and cardiovascular diseases. Dtsch Med Wochenschr 2005, 130:2904-2909.

18. Nageswara RM, Aleksandr V, Marschall SR: Oxidative stress and vascular disease. Arterioscler Thromb Vasc Biol 2005, 25:29-38.

19. Antelava NA, Pachkoriia KZ, Kezeli TD, Nikuradze NS, Shamkulashvili GG: Major pathogenic links of atherosclerosis. Georgian Med News 2005, 128:72-79.

20. Yokoyama M: Oxidative stress and atherosclerosis. Curr Opin Pharmacol 2004, 4:110-115.
21. Wei W, Wu XM, Li YJ: Behavioral pharmacology experiment method: methodology of pharmacological experiment: chapter 19. In 4th edition Edited by Ma Y, Zhou WX, Zhang YX. China: Pepole's Medical Publishing House; 2010,635-636.

22. Guo DY, Chen TY, Li B, Zhang L, Li YL, Li L: Behavioral comparison among the rats at different age in water maze and open field. Acta Laboratorium Animalis Scientia Sinica 1998, 6:19-23.

doi:10.1186/1476-511X-12-118

Cite this article as: Gao et al:: Acute stress show great influences on liver function and the expression of hepatic genes associated with lipid metabolism in rats. Lipids in Health and Disease 2013 12:118.

\section{Submit your next manuscript to BioMed Central and take full advantage of:}

- Convenient online submission

- Thorough peer review

- No space constraints or color figure charges

- Immediate publication on acceptance

- Inclusion in PubMed, CAS, Scopus and Google Scholar

- Research which is freely available for redistribution 